\title{
Dispersion of $\mathrm{CaCO}_{3}$ nanoparticles by sonication and surfactant treatment for application in fly ash-cement systems
}

\author{
Shiho Kawashima $\cdot$ Jung-Woo Ted Seo $\cdot$ \\ David Corr • Mark C. Hersam - Surendra P. Shah
}

Received: 24 December 2012/ Accepted: 28 May 2013

(C) RILEM 2013

\begin{abstract}
This research aims to offset the negative effects of fly ash on the early-age properties of cementitious materials with the use of calcium carbonate $\left(\mathrm{CaCO}_{3}\right)$ nanoparticles. The main focus is to enhance the effect of the nanoparticles by improving dispersion through ultrasonication and use of surfactants. $\mathrm{CaCO}_{3}$ aqueous suspensions with various surfactant types and concentrations are prepared and subjected to different sonication protocols (varying duration and amplitude). Dispersion and stability are quantitatively measured by comparing their absorbance spectra through spectrophotometry and qualitatively evaluated through SEM imaging. The effectiveness of sonicated $\mathrm{CaCO}_{3}$ nanoparticle additions in accelerating setting and improving early-age compressive strength gain of fly ash-cement pastes is investigated. The sonication protocol is optimized and
\end{abstract}

S. Kawashima ( $\square)$

Department of Civil Engineering and Engineering

Mechanics, Columbia University, 500 West 120th Street,

616 Mudd, New York, NY 10027, USA

e-mail: s-kawashima@columbia.edu

S. Kawashima · D. Corr · S. P. Shah

Department of Civil and Environmental Engineering, Northwestern University, 2145 Sheridan Road, Evanston, IL 60208, USA

J.-W. T. Seo - M. C. Hersam

Department of Materials Science and Engineering,

Northwestern University, 2220 Campus Drive, Cook Hall,

Evanston, IL 60208, USA the most effective dispersion is achieved with polycarboxylate-based superplasticizer. Good agreement is found between the dispersion measurements and mechanical performance.

Keywords Calcium carbonate $\cdot$ Nanoparticles · Dispersion - Sonication · Fly ash .

Cement and Concrete

\section{Introduction}

With increasing societal demands to make concrete a more sustainable infrastructural material, the replacement of cement with supplementary cementitious materials (SCMs) has been widely recognized as a viable solution. One such SCM is fly ash, a coal combustion byproduct that can introduce advantages to the rheological and long-term properties of concrete. However, it is known to negatively impact the earlyage properties. Due to the pozzolanic nature of fly ash, a secondary reaction that can only occur after primary cement hydration, it significantly slows setting and early-age compressive strength gain, both of which slow construction. To further promote the use of fly ash, there is a need to offset these negative effects.

The use of nanoparticles has been demonstrated to activate cement hydration, which can help to accelerate the formation of calcium hydroxide $(\mathrm{CH})$ and 
subsequently initiate the pozzolanic activity of fly ash. Thus far, most studies have focused on nanosilica and demonstrated its effectiveness in improving the earlyage properties [26, 39, 45]. However, a recent study by Hou et al. [23] showed that it may have negative effects on the later-age properties. Also being a pozzolan, nanosilica is in direct competition with fly ash for $\mathrm{CH}$. Furthermore, due to the fineness of nanosilica, it exhibits higher reactivity, which results in high rate of $\mathrm{CH}$ consumption at early ages. Although this has good implications on the early-age properties, it leaves less $\mathrm{CH}$ for fly ash to react with later on to sustain long-term strength gain. As the suitability of nanosilica for fly ash-cement systems remains inconclusive, it is necessary to look for alternative types of nanoparticles for the application of activating fly ash-cement systems.

Limestone, or calcium carbonate $\left(\mathrm{CaCO}_{3}\right)$, is primarily used as a filler to improve rheological properties in its powder form; however it has also been shown to affect cement hydration. Studies have shown that $\mathrm{CaCO}_{3}$ can accelerate early-age hydration, provide nucleation sites for $\mathrm{C}-\mathrm{S}-\mathrm{H}$ (and perhaps $\mathrm{CH}$ ), and react with calcium aluminates $\left(\mathrm{C}_{3} \mathrm{~A}\right)$ and tricalcium silicate $\left(\mathrm{C}_{3} \mathrm{~S}\right)$ to produce calcium carboaluminates and calcium carbosilicate hydrates, respectively $[5,7,15$, $22,24,29,30,34,42,54]$. Given these effects, $\mathrm{CaCO}_{3}$ is considered to be a good candidate for inclusion in fly ash-cement systems. Thus far the focus has mainly been on micro-sized limestone powder. $\mathrm{NanoCaCO}_{3}$ has not been as widely investigated but studies have shown the potential of the material in offsetting the negative effects of fly ash on the early-age properties, even at high replacement rates [5, 8, 20, 36, 47, 48].

From a practical standpoint, the effective use of nanoparticles relies on the ability to achieve uniform and stable dispersions. This is expected to enhance their performance and reduce the necessary amount of materials for applications, which ensures its economic viability. One of the major challenges in using nanoparticles over microparticles is that uniform dispersion becomes significantly more challenging. Due to their high surface area and energy, nanoparticles experience increased surface interactions and are more susceptible to particle adhesion upon direct contact by van der Waals, electrostatic and magnetic forces. Although a critical issue, dispersion is not addressed in most studies regarding nanoparticles in cement and concrete research, as reported in a paper by
Bagheri et al. [4]. In this study, we explore methods to obtain well-dispersed, stable $\mathrm{CaCO}_{3}$ nanoparticles in an aqueous medium through sonication and the use of surfactants. Previous work by the authors has demonstrated the potential of the method [28]. Various sonication protocols and surfactant types are investigated. Dispersion quality and stability are quantitatively measured through methods that utilize optical absorbance spectroscopy. They are also qualitatively confirmed through SEM imaging. Degree of dispersion is then tied to the influence of the nanoparticles on the early-age properties of fly ash-cement pastes (CPs).

\section{Materials and experimental methods}

\subsection{Materials}

Type I Portland cement and tap water are used in all samples. Type F fly ash is used in select mixes. The chemical composition of the cement and fly ash are shown in Table 1. The $\mathrm{CaCO}_{3}$ nanoparticles (from Reade Advanced Materials) are in dry powder form and have a particle size range of 15-40 nm according to the manufacturer. From the SEM image, shown in Fig. 1, it is apparent that the as-received material consists of aggregates on the micron scale.

A number of surfactants are tested to determine their suitability to disperse and stabilize the $\mathrm{CaCO}_{3}$ nanoparticles in aqueous solution. They are listed in Table 2 and their chemical structures are shown in Fig. 2. Two commercially based superplasticizers are tested: one naphthalene-based (WR Grace) and one polycarboxylate based (BASF). Two anionic,

Table 1 Chemical composition of cement and fly ash

\begin{tabular}{lll}
\hline Materials & Type I cement & Type F fly ash \\
\hline $\mathrm{SiO}_{2}$ & 20.2 & 46 \\
$\mathrm{Al}_{2} \mathrm{O}_{3}$ & 4.7 & 17.8 \\
$\mathrm{Fe}_{2} \mathrm{O}_{3}$ & 3.3 & 18.2 \\
$\mathrm{SO}_{3}$ & 3.3 & 2.59 \\
$\mathrm{CaO}$ & 62.9 & 8.4 \\
$\mathrm{MgO}$ & 2.7 & 0.95 \\
$\mathrm{Na} 2$ & $/$ & 0.59 \\
$\mathrm{~K}_{2} \mathrm{O}$ & $/$ & 2.16 \\
$\mathrm{LOI}$ & 1.1 & 1.49 \\
Total & 98.2 & 98.2 \\
\hline
\end{tabular}




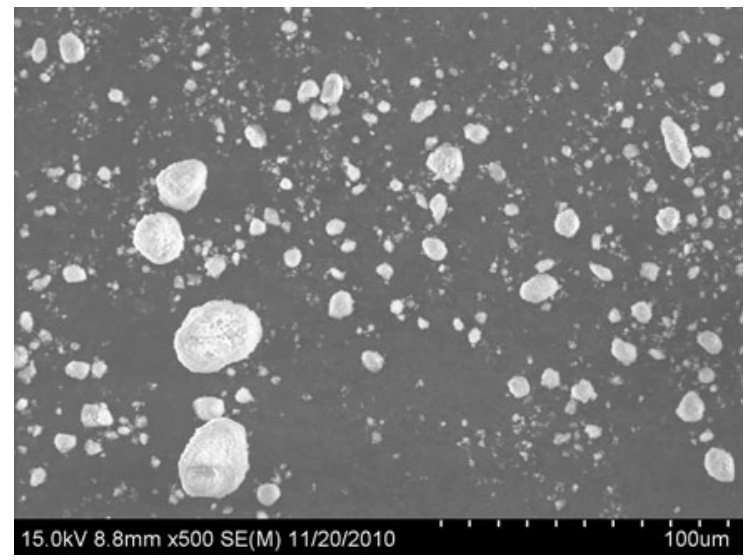

Fig. 1 SEM image of aggregated $\mathrm{CaCO}_{3}$ nanoparticles in the dry powder form [27]

amphiphilic surfactants are tested: sodium cholate (SC) (Sigma Aldrich) and sodium dodecyl sulfate (SDS) (Fisher Scientific), respectively. And three variants of a nonionic, amphiphilic block copolymer with polypropylene oxide and polyethylene oxide chains (T1109, T904, T908) are tested, from BASF. Cationic surfactants are not tested, as they have been found to flocculate $\mathrm{CaCO}_{3}[25,38,41]$. SC and SDS are chosen here as they have been widely used as surfactants for carbon-based nanomaterials, where they demonstrate exceptional dispersion stability and ability to isolate a variety of low-dimensional structures including nanotubes and nanosheets $[2,17,18$, 50]. The Tetronic block copolymers are also popular dispersants for such carbon systems [1, 49] and will be studied to explore their suitability with non-carbon nanoparticles as surfactants. Tetronics block copolymers follow a naming convention where the last digit of their name multiplied by 10 indicates the percentage by weight of their hydrophilic chains, while the

Table 2 List of surfactants

\begin{tabular}{ll}
\hline Surfactant & Type \\
\hline N & Naphthalene-based superplasticizer \\
PC & Polycarboxylate-based superplasticizer \\
SC & Sodium cholate \\
SDS & Sodium dodecyl sulfate \\
T1109 & Variants of a nonionic, amphiphilic block \\
T904 & copolymers with polypropylene oxide \\
T908 & and polyethylene oxide chains \\
\hline
\end{tabular}

earlier digits multiplied by 45 provide the approximate molecular weight of the hydroblock segment.

\subsection{Experimental methods}

\subsubsection{Dispersion in aqueous solution}

2.2.1.1 Horn sonication To break up the aggregated $\mathrm{CaCO}_{3}$ nanoparticles in aqueous solution, ultrasonication (Fisher Scientific model 500 Sonic Dismembrator) is implemented. Each constituent (i.e. nanoCaCO water, and surfactant) is measured directly into a metal cup using a precision scale. Then, the sample is placed in the holder and lifted up so the tip of the horn is submerged in the solution but not touching the base of the metal cup. An ice bath is placed on a holder, as well, and raised up to surround the sample to prevent overheating and evaporation during the sonication process. The sonic dismembrator is programmed to apply a constant sonication for a set duration and amplitude.

2.2.1.2 Bath sonication The maximum capacity for horn sonication (HS) is approximately $200 \mathrm{~mL}$. In order to utilize these suspensions for preparing paste samples for mechanical testing, they are scaled up by bath sonication (Bransonic 3510 tabletop ultrasonic cleaner). The bath sonicator used in this study allows for up to $2 \mathrm{~L}$ of solution to be prepared. The samples are placed in a holder within the bath to prevent direct contact with the walls of the sonicator. Sonication is performed at room temperature. The water is degassed for $10 \mathrm{~min}$ prior to sonication and the initial water level is kept consistent for all samples.

\subsubsection{Evaluating dispersion}

To obtain a quantitative measure of dispersion, the absorbance spectra of nanoCaCO 3 aqueous suspensions are measured by a spectrophotometer (Varian Cary 5000). According to Beer's law, the optical absorbance of solution-phase suspension exhibits a linear relationship with the concentration of substance. It is expected that nanoparticles that are dispersed and stabilized will remain in suspension. On the other hand, any aggregated particles will settle to the bottom. By noting the decrease in optical density of samples, it is possible to deduce the extent of sedimentation and thus the quality of dispersions prepared via various sonication protocols and surfactants. 
(a)

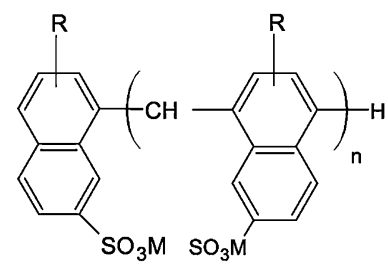

(b)

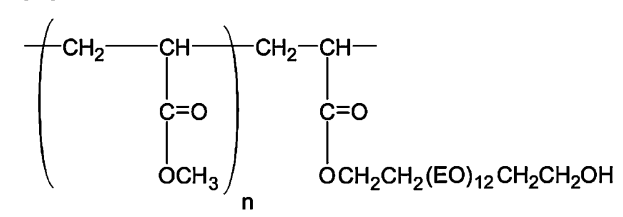

(c)

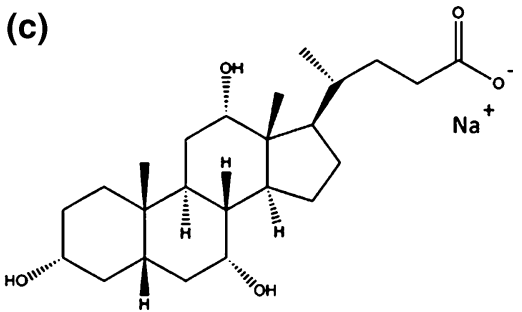

(d)<smiles>CCCCCCCCCCCCCOP(=O)(O)OC</smiles>

(e)

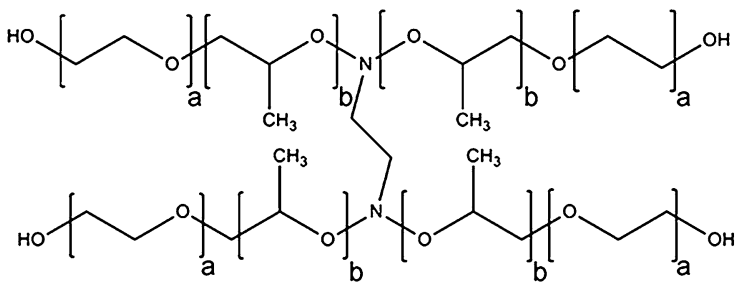

Fig. 2 Chemical structures of surfactants: a naphthalene-based superplasticizer and b polycarboxylate-based superplasticizer [35]; c SC, d SDS, and e Tetronic

In order to ensure that the spectra measured are only of the suspended nanoparticles and not the surfactant, baseline readings are taken before each set of measurements. All spectra readings are taken between wavelengths of $380-800 \mathrm{~nm}$ and the absorbance at $550 \mathrm{~nm}$ is compared. Tested suspensions are diluted with surfactant solution to prevent over-saturation of the absorbance from samples with nanoparticles: $50 \mu \mathrm{L}_{\text {of }} \mathrm{CaCO}_{3}$ suspension is placed in a cuvette and then diluted with $800 \mu \mathrm{L}$ of surfactant solution. Before each reading, the cuvettes are agitated using a vortex mixer/shaker to obtain a uniform sample. In all cases, three samples are measured and the average is taken to be the representative absorbance. Detailed information on the procedures for all test methods can be found elsewhere [27].

\subsubsection{Centrifugal method The absorbance spectra} are measured for the supernatant of centrifuged nanoCaCO $\mathrm{CO}_{3}$ suspensions and suspensions obtained immediately after sonication. The latter serves as a reference point, at which the majority of nanoparticles are expected to be suspended momentarily. During centrifugation any undispersed, aggregated particles will sediment to the bottom while the dispersed nanoparticles will remain suspended in the supernatant. By comparing the absorbance before and after centrifugation, a measure of degree of dispersion after sonication is obtained.
Suspensions are transferred to conical micro-centrifuge tubes, then centrifuged at 5,000 rpm for $2 \mathrm{~min}$. After centrifugation, $50 \mu \mathrm{L}$ of the supernatant is decanted, placed in a cuvette and diluted with $800 \mu \mathrm{L}$ of surfactant solution. Similar steps are followed for the reference samples obtained immediately after sonication.

\subsubsection{Sedimentation over time The centrifugation} method subjects the suspensions to high centripetal force over a short period of time. Although this provides a quick measure of degree of dispersion, it is of interest to measure the sedimentation of nanoparticles over time when the suspension is left at rest. During sonication the nanoparticles may be broken up into primary particles or primary aggregates but it is possible that they will reaggregate over time. To measure the rate at which this is occurring, the evolution of sedimentation is measured.

The change in absorbance of the suspension is monitored up to $24 \mathrm{~h}$. Immediately after sonication, 15 micro-centrifuge tubes are each filled with $1.5 \mathrm{~mL}$ of the suspension with a Pasteur pipette. Then at 0, 0.5, 3, 6 and $24 \mathrm{~h}, 1.0 \mathrm{~mL}$ of supernatant from three samples are decanted and transferred to three new centrifuge tubes. Cuvettes are filled with $50 \mu \mathrm{L}$ of the decanted solution and $800 \mu \mathrm{L}$ of surfactant solution, then tested. Monitoring the decrease in absorbance over time gives 
a measure of the rate of reaggregation when the suspension is left at rest.

\subsubsection{Early-age properties}

The setting and compressive strength gain of CPs with a $30 \%$ fly ash replacement by mass and a w/b ratio of 0.35 are measured. Pastes are prepared in a small upright mixer for $10 \mathrm{~min}$ at the lowest setting. When nanoCaCO $\mathrm{C}_{3}$ is added, it is introduced as an aqueous suspension and the water content of the suspension serves as the mixing water for the sample. This suspension is added to the dry ingredients, i.e. cement and fly ash, during mixing.

Setting time of pastes are measured using the Vicat needle test based on ASTM standard C191-08. And compressive strength of cubic samples [50 mm] at 1 , 3 , and 7 are measured in accordance to ASTM standard C109/C109 M-08. 3 and 7 days samples are cured in water at room temperature until testing. Three samples are tested for each mix, from which the average is taken to be the representative measurement.

\subsubsection{Scanning electron microscopy (SEM)}

Hitachi S-4800 FE-SEM equipped with energy dispersive spectroscopy (EDS) is used to characterize the state of dispersion of the $\mathrm{CaCO}_{3}$ nanoparticles. For nanoCaCO${ }_{3}$ aqueous suspensions, a drop is placed on a sample holder and allowed to dry under ambient conditions for at least $2 \mathrm{~h}$. For hardened CP samples, a fractured piece obtained after compressive strength testing is soaked in acetone to stop hydration. All samples are coated with $20-25 \mathrm{~nm}$ of gold. The accelerating voltage and current are $15-20 \mathrm{kV}$ and 10-20 $\mu \mathrm{A}$, respectively. The SEM is set so that the upper detector collects the secondary electrons.

\section{Experimental results and discussion}

\subsection{Dispersion in aqueous solution}

\subsubsection{Centrifugal method}

3.1.1.1 Influence of surfactant type To determine the effect of surfactant type, all suspensions are subjected to the same sonication protocol: HS for $3 \mathrm{~h}$ at $40 \%$ amplitude, the total energy of which is
356,400 J. The results are shown in Table 3. Sedimentation is defined as the following:

$S=100 \%-\frac{\operatorname{Abs}_{C}}{\mathrm{Abs}_{t=0}} \cdot 100 \%$

where $\mathrm{Abs}_{t=0}$ is absorbance at $550 \mathrm{~nm}$ for the suspension immediately after sonication (reading taken within 5 min after end of sonication) and $\mathrm{Abs}_{C}$ is absorbance at $550 \mathrm{~nm}$ of the supernatant after centrifugation. In the case of no surfactant, all of the nanoparticles settle to the bottom. It is apparent that although $\mathrm{CaCO}_{3}$ is considered to be hydrophilic, the degree of hydrophilicity is not high enough to prevent the large particles from reaggregating. Therefore, this proves that the use of surfactants is necessary for stabilization.

A range of dosages for each surfactant is tested (7-200\% surfactant by mass of nanoCaCO $\mathrm{Ca}_{3}$ ) but only select results, which are considered to be representative, are included herein. It is apparent from Table 3 that among the surfactants presented in this study, PC showed the highest effectiveness where almost $10 \%$ of the $\mathrm{CaCO}_{3}$ nanoparticles are retained after centrifugation. SC and SDS are considered to be conventional anionic surfactants that exhibit amphiphilic properties, where SC has a planar structure while SDS is linearly shaped. Adsorption of the surfactant onto the $\mathrm{CaCO}_{3}$ is expected to occur by both electrostatic interaction with the $\mathrm{Ca}^{2+}$ sites and hydrophobic bonding. Although anionic surfactants have been found to disperse micro-sized limestone particles $[40,46]$, it is found here that they are not sufficient

Table 3 Influence of surfactant type on dispersion: all suspensions contain $3 \mathrm{~g} \mathrm{CaCO}_{3}$ nanoparticles and $129 \mathrm{~g}$ of water [27]

\begin{tabular}{lll}
\hline Surfactant type & Surfactant $(\mathrm{g})$ & Sedimentation $(\%)$ \\
\hline None & 0 & - \\
$\mathrm{N}$ & 1 & 97.7 \\
$\mathrm{PC}$ & 1 & 92.1 \\
$\mathrm{SC}$ & 1.3 & - \\
SDS & 1.3 & - \\
T1109 & 3 & - \\
T904 & 3 & - \\
T908 & 3 & - \\
\hline
\end{tabular}

Sedimentation marked with a "_" indicate supernatants which run clear after centrifugation, i.e. all the nanoparticles in the suspension settle to the bottom 
in the case of nano-sized particles, where suspensions remained clearly segregated.

Results indicate that PC is the most effective. Although the exact formulation is not known, generally PC superplasticizers are comb polymers consisting of an anionic backbone with carboxylic acid groups and grafted side chains mainly composed of hydrophilic polyethylene oxide (PEO) units. The improvement in nanoCaCO${ }_{3}$ dispersion by $\mathrm{PC}$ may be attributed to both electrostatic and steric hindrance: the anionic backbone adsorbs onto the calcium and the PEO chains extend out between adjacent nanoparticles. Polyacrylates, or acrylic acids (sodium [3, 14] and ammonium [19]), have been found to be effective dispersants for $\mathrm{CaCO}_{3}$ powders, including nanoparticles [11], as they readily adsorb onto their surface. Acrylic acids are a type of carboxylic acid. Therefore the carboxylate (an ether of carboxylic acid) backbone of the superplasticizer should be adsorbing onto the nanoCaCO $\mathrm{C}_{3}$ in a similar manner.

The naphthalene-based superplasticizer did not yield good dispersions. While PCs disperse through electrostatic and steric repulsion with the anionic backbone and PEO chains, respectively, naphthalenebased superplasticizers act only through electrostatic repulsion $[6,9,10]$. Similarly to the SC and SDS, this is not sufficient for dispersing the $\mathrm{CaCO}_{3}$ nanoparticles in an aqueous solution.

Three variants of a non-ionic, amphiphilic block copolymer are studied. They are commercially available poloxamine: X-shaped and formed by four polypropylene oxide (PPO)-PEO chains bonded to an ethylene diamine central group, where the molecular weight of the PPO and PEO chains can be changed. Seo et al. [49] found that the molecular weight of each of the chains had a significant influence on the dispersability of graphene in aqueous solution. None of the variants, however, are effective in dispersing the nanoCaCO${ }_{3}$. This is likely due to the difference in surface properties between graphene and $\mathrm{CaCO}_{3}$.

\subsubsection{Influence of sonication amplitude and} duration To verify that sonication is aiding the dispersion process, the absorbance of the suspension treated with PC and prepared by mechanical stirring is evaluated. Dispersion is compared for suspensions that are sonicated for $3 \mathrm{~h}$ at $40 \%$ amplitude and mechanically stirred for $3 \mathrm{~h}$. Both suspensions are treated with $\mathrm{PC}$ at a concentration of $33 \%$ by mass of nanoCaCO $\mathrm{C}_{3}$. It is found that the sedimentation of the mechanically stirred suspension is $99.1 \%$, compared to $92.1 \%$ for the sonicated. This verifies that sonication is enhancing the dispersion process by physically breaking up the aggregates while PC is acting to stabilize the nanoparticles once they are dispersed.

The level of dispersion for suspensions treated with $33 \%$ PC by mass of nanoCaCO $\mathrm{CO}_{3}$ subjected to ultrasonication for different durations and amplitudes are presented in Table 4. This is to determine the protocol that achieves the best dispersion while limiting the amount of energy required for processing the nanoparticles. Evaluating the influence of duration, the sedimentation between 1,3 , and $6 \mathrm{~h}$ at a fixed amplitude of $40 \%$ are compared. It is found that dispersion improves with duration. However, the rate of decrease in sedimentation goes down-the decrease from 1 to $3 \mathrm{~h}$ is greater than that from 3 to $6 \mathrm{~h}$.

To determine the effect of amplitude, samples treated with amplitudes of 35,40 , and $50 \%$ for a fixed duration of $3 \mathrm{~h}$ are compared. Decreasing the amplitude from 40 to $35 \%$ results in a notable increase in sedimentation from 92.1 to $98.9 \%$. On the other hand, an increase from 40 to $50 \%$ does not result in any apparent improvement. There appears to be a threshold for amplitude-beyond $40 \%$ there is no significant improvement in dispersion for the given sonication setup. Regarding duration, although longer duration shows an improvement in level of dispersion, the energy demand increases disproportionally to it. It is likely that the sedimentation is tied to issues of reaggregation, as observed in another study where the aggregate size of sonicated nanoparticles increased from $500 \mathrm{~nm}$ to $3-4 \mu \mathrm{m}$ [37].

Table 4 Influence of time and amplitude of sonication on dispersion: all suspensions contain $1 \mathrm{~g} \mathrm{PC}, 3 \mathrm{~g} \mathrm{CaCO}_{3}$ nanoparticles, and $129 \mathrm{~g}$ water [27]

\begin{tabular}{llll}
\hline Time (h) & Amplitude $(\%)$ & Energy $(\mathrm{J})$ & Sedimentation $(\%)$ \\
\hline 1 & 40 & 118,800 & 95.1 \\
3 & 40 & 356,400 & 92.1 \\
6 & 40 & 712,800 & 90.2 \\
3 & 50 & 475,200 & 92.9 \\
3 & 35 & 311,850 & 98.9 \\
6 & 30 & 534,600 & 96.5 \\
\hline
\end{tabular}


There have been some reported cases where high energy input induced reaggregation [32]. In order to verify this, in the present study a sample is prepared by applying lower amplitude for a longer duration (6 h at $30 \%$ ). However this is not found to improve dispersion. The results suggest that instead of improving dispersion through modifying the sonication protocol, which will affect the size of aggregates but not necessarily prevent reaggregation, it is more efficient to find a more suitable surfactant that will stabilize the particles once they are dispersed.

3.1.1.3 Influence of concentration The influence of surfactant concentration is determined. Based on the previously discussed results, samples are treated with PC and sonicated for $3 \mathrm{~h}$ at $40 \%$. $\mathrm{NanoCaCO}_{3}$ suspensions treated with different concentrations are tested and the results are shown in Table 5. Sedimentation of suspensions treated with 17 and $33 \%$ PC are comparable, whereas at $7 \%$ it increases substantially.

\subsubsection{Sedimentation over time}

The sedimentation over time at rest of PC treated nanoparticles is measured. The evolution of sedimentation is recorded for suspensions prepared by horn and bath sonication. All suspensions are treated with $\mathrm{PC}$ at a concentration of $33 \%$ by mass of nanoCaCO $\mathrm{CO}_{3}$. For the suspension prepared by HS, it is subjected to sonication for $3 \mathrm{~h}$ at $40 \%$ amplitude. Bath sonication is a much less energy intensive method and thereby requires a longer duration. Suspensions prepared by bath sonication for 6 and $9 \mathrm{~h}$ (BS6 and BS9) are compared. The absorbance from 380 to $800 \mathrm{~nm}$ of each sample immediately after sonication is shown in Fig. 3 considered as the initial reading. As shown, the optical density of each sample is similar, indicating that the initial degree of dispersion achieved is comparable between all the suspensions. The

Table 5 Influence of PC addition on dispersion: all suspensions horn sonicated for $3 \mathrm{~h}$ at $40 \%$ amplitude [27]

\begin{tabular}{ll}
\hline $\mathrm{PC}(\%$ by mass of nanoCaCO & \\
\hline 7 & Sedimentation $(\%)$ \\
17 & 99.9 \\
33 & 92.3 \\
\hline
\end{tabular}

absorbance at $550 \mathrm{~nm}$ is normalized and plotted as amount retained in Fig. 4. The sample that is horn sonicated exhibits a lower sedimentation rate compared to both bath sonicated samples. Over the first $30 \mathrm{~min}$, retention drops $10 \%$ for BS6 and BS9 while it drops less than $5 \%$ for HS. After $30 \mathrm{~min}$, the rate of sedimentation is about the same between all the samples up to $3 \mathrm{~h}$. At $24 \mathrm{~h}$, BS6 is found to experience the highest sedimentation.

Although PCs are found to be the best dispersant in the present study, the percent retained is still relatively low: approximately $10 \%$ as measured by the centrifugal method and $60 \%$ at $24 \mathrm{~h}$ by the sedimentation method. Although PC is expected to adsorb onto

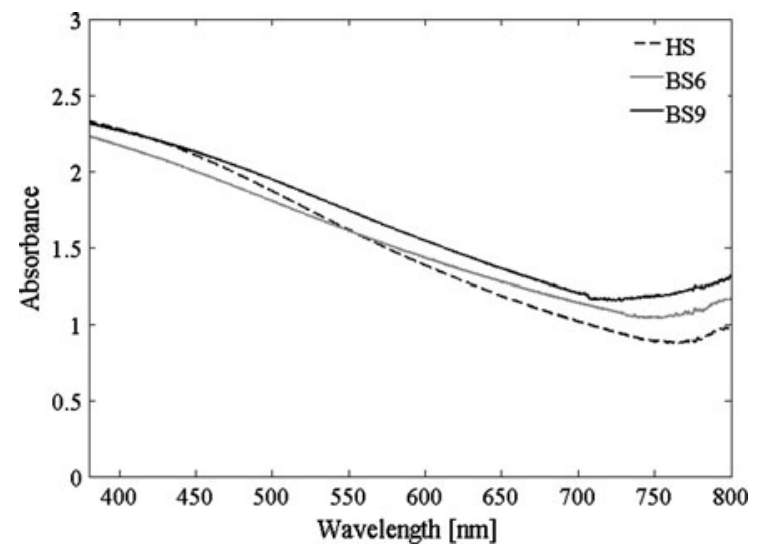

Fig. 3 Absorbance spectra of suspensions immediately after sonication: horn sonicated (HS), bath sonicated for $6 \mathrm{~h}$ (BS6) and $9 \mathrm{~h}$ (BS9) [27]

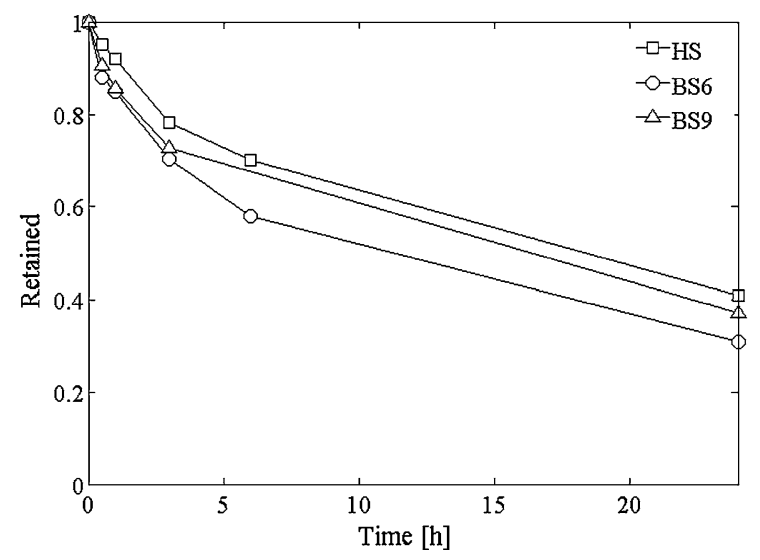

Fig. $4 \mathrm{NanoCaCO}_{3}$ retained in suspension (normalized) over time: horn sonicated (HS), bath sonicated for $6 \mathrm{~h}$ (BS6) and $9 \mathrm{~h}$ (BS9) [27] 
calcium, the fine size of the nanoparticles may be a critical factor. The high surface area significantly increases adsorption demand and uneven adsorption will lead to aggregation. Further, there are cases where poor adsorption of PC-based superplasticizers on calcite has been reported $[12,13]$.

Although PCs are common concrete admixtures, the behavior of PC-based superplasticizers in cementitous systems is complex and the adsorption properties are still not well understood [16, 21, 43, 56]. However, PCs are designed so that the backbone adsorbs onto the various phases of cement and hydrates (most containing calcium) and the PEO "teeth" remain in pore solution. Researchers have found that the carboxylic group preferentially binds to the $\mathrm{Ca}^{2+}$ sites $[44,51$, 52]. Similar adsorption behavior is expected on the nanoCaCO${ }_{3}$. However, it may be possible to improve dispersion by trying different architectures of the superplasticizer. The chemical structure of PC-based superplasticizers can be varied by changing its side chain length and density, which will effectively change its anionic charge density. These parameters can have a significant effect on their adsorption and dispersing capabilities [12, 31, 33, 53, 55]. Since commercially available superplasticizers like the one used in the present study are designed to disperse cement particles and hydrates, their architecture is likely not the most suitable for nanoCaCO 3 . It is necessary to synthesize the PCs and systematically vary chain lengths and densities to determine what is most suitable for nanoCaCO${ }_{3}$. This was not within the scope of the present study but could be the topic of future work.

\subsubsection{SEM imaging}

As measured through absorbance spectra, results show that nanoparticles treated with PC and subjected to sonication lead to improved dispersion. To complement these results, suspensions prepared by mechanical stirring, HS and bath sonication are observed under SEM. Since the samples need to be dried for SEM imaging, it does not capture their true state in suspension. Despite this limitation, it can still provide information on the state of aggregation for comparative purposes.

The results are shown in Fig. 5. It is apparent that nanoparticles that are stirred still remain highly aggregated, Fig. 5a, with aggregates exceeding $20 \mu$.
This is similar to the state of the nanoparticles in the dry powder form, Fig. 1. On the other hand, with sonication the particles are in a plane and no large aggregates are present, as shown in Fig. 5b. This supports that the method is aiding the dispersion process by breaking up aggregates.

\subsection{Early-age properties}

The sedimentation that occurs over a long term is not ideal. However, for the purposes of evaluating the influence of dispersed nanoCaCO 3 on the properties of fly-ash cementitious materials, the level of dispersion at the time of sample preparation is the most critical. It is possible to cast cementitious samples for mechanical testing within $1 \mathrm{~h}$ after sonication. Therefore the degree of dispersion over $1 \mathrm{~h}$ is important. According to Fig. 4, at least $85 \%$ is retained after $1 \mathrm{~h}$ and $70 \%$ after $3 \mathrm{~h}$ for all samples. It can thereby be assumed that

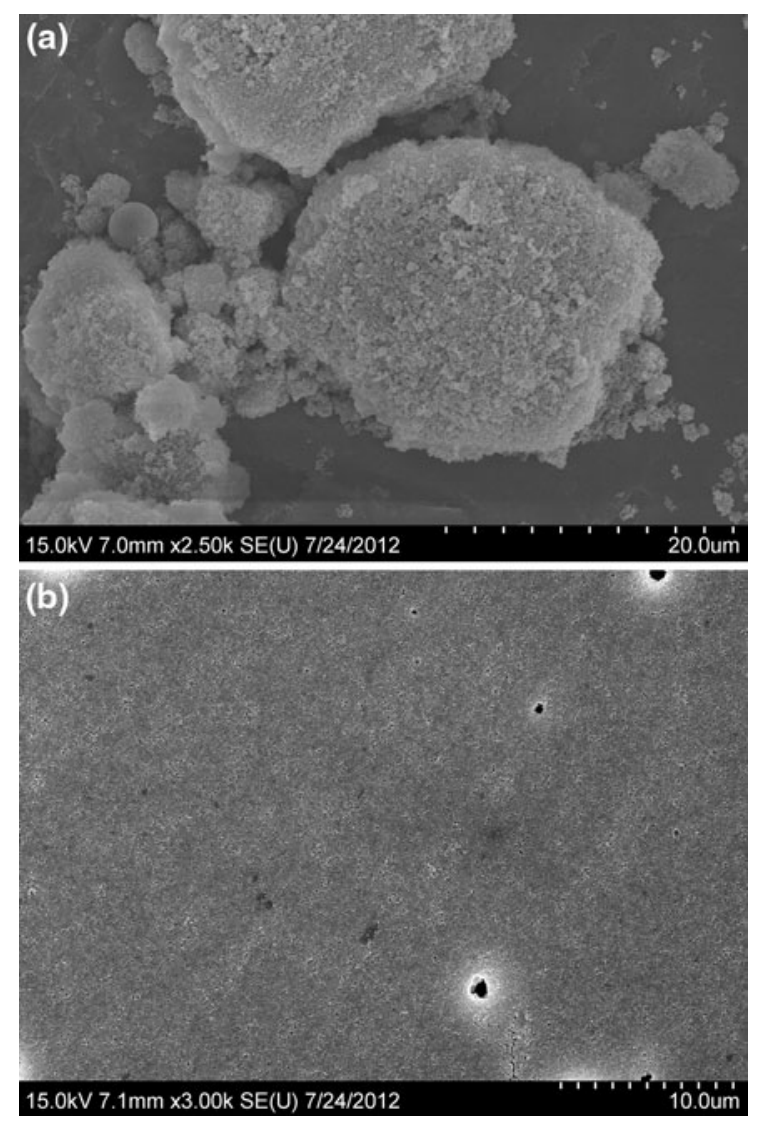

Fig. 5 SEM images of nanoCaCO 3 particles treated with PC ( $0.33 \%$ by mass) and prepared by a mechanical stirring and b bath sonication [27] 
if the samples are cast within this time frame, the majority of the nanoparticles are still dispersed and suspended. Additionally, the nanoparticles can be sonicated again to re-disperse in solution moments before sample preparation, which enhances the economic viability of such a product.

\subsubsection{Setting}

The setting times of CPs with $30 \%$ fly ash replacement are compared against that of plain CP. $30 \%$ fly ash-CPs without nanoCaCO 3 (30FA) and with $1 \%$ nanoCaCO $\mathrm{CaC}_{3}(1 \mathrm{nLS})$ dispersed either by stirring or ultrasonication are compared. For both dispersion methods, the nanoparticles are treated with PC, added at a concentration of $33 \%$ by mass of nanoCaCO $\mathrm{Cl}_{3}$. All samples (including those without any nanoparticles) have a $0.33 \%$ PC addition by mass of binder.

The results are shown in Fig. 6. Two samples are tested for each mix. The replacement of $30 \%$ of cement with fly ash delays initial and final set by approximately $2 \mathrm{~h}$. With the addition of $1 \%$ nanoC$\mathrm{aCO}_{3}$ in both cases, stirred and sonicated, setting is accelerated. With the stirred nanoparticles, initial and final set are shortened by approximately $1 \mathrm{~h}$. There is greater improvement with the sonicated nanoparticles, where set time is accelerated by more than $1.5 \mathrm{~h}$. This indicates that sonication enhances the effect of the nanoparticles. This is likely due to the seeding effect, which increases with improved dispersion. Welldispersed nanoparticles can accelerate the start of the

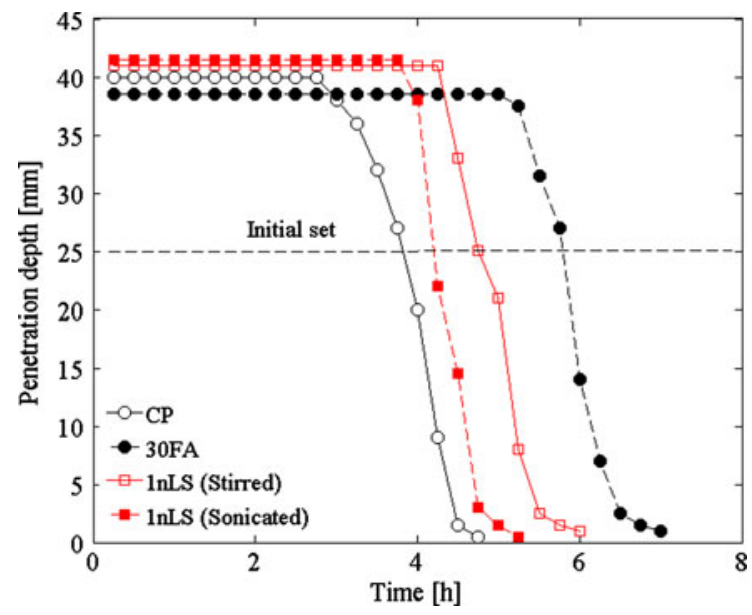

Fig. 6 Setting time: plain cement paste (CP) and $30 \%$ fly ashcement pastes with $0 \%$ (30FA) and $1 \%$ nanoCaCO $\mathrm{CaCO}_{3}(1 \mathrm{nLS})$ addition [27] main hydration phase and increase the number of nucleation sites from which $\mathrm{C}-\mathrm{S}-\mathrm{H}$ can grow. The latter allows the microstructure to become percolated more rapidly and subsequently lead to faster setting. There may also be a filler effect, where the nanoparticles are providing more contact points within the structure, therefore leading to an interconnected network more rapidly.

\subsubsection{Compressive strength gain}

The compressive strengths at 1,3 and 7 days are compared for $30 \%$ fly ash-CPs with PC-treated, sonicated nanoCaCO${ }_{3}$. Mixes with nanoCaCO${ }_{3}$ additions of $1,2.5$ and $5 \%$ (1nLS, 2.5nLS, and 5nLS) by mass of binder are compared. The mix designs are shown in Table 6. In mixes $2.5 \mathrm{nLS}$ and $5 \mathrm{nLS}$, the PC concentration is 16.5 and $8.3 \%$ by mass of nanoC$\mathrm{aCO}_{3}$, respectively. (This corresponds to $0.4 \%$ by mass of binder for both cases.) According to the results of the dispersion tests, see Table 5, a PC concentration of $16.5 \%$ still returns sufficient dispersion while at $7 \%$ the degree of dispersion goes down significantly. However, the addition of PC allowable is limited by the rheological properties of the CPs, where increasing the PC addition in the pastes any more than $0.4 \%$ would lead to segregation and bleeding. At $1 \%$ nanoCaCO $\mathrm{Ca}_{3}$ addition the suspensions are horn sonicated for $3 \mathrm{~h}$ at $40 \%$ amplitude while at 2.5 and $5 \%$ the suspensions are bath sonicated for $9 \mathrm{~h}$.

In Fig. 7, the influence of $30 \%$ fly ash replacement and $1 \%$ nanoCaCO${ }_{3}$ are shown. The replacement of $30 \%$ of cement with fly ash leads to a decrease in strength gain at all ages compared to the plain $\mathrm{CP}$. With the addition of $1 \%$ nanoCaCO 3 , there is little influence at 1 and 3 days, as shown in the strengths for both mixes 1nLS (sonicated) and 1nLS (stirred). However, by

Table 6 Mix design for compressive strength paste samples

\begin{tabular}{llclll}
\hline Mix & $\begin{array}{l}\text { Cement } \\
(\mathrm{g})\end{array}$ & $\begin{array}{l}\text { Fly ash } \\
(\mathrm{g})\end{array}$ & $\begin{array}{l}\text { Water } \\
(\mathrm{g})\end{array}$ & $\begin{array}{l}\mathrm{NanoCaCO}_{3} \\
(\mathrm{~g})\end{array}$ & $\begin{array}{l}\mathrm{PC} \\
(\mathrm{g})\end{array}$ \\
\hline $\mathrm{CP}$ & 750 & 0 & 262.5 & 0 & 2.5 \\
30FA & 525 & 225 & 262.5 & 0 & 2.5 \\
1nLS & 525 & 225 & 262.5 & 7.5 & 2.5 \\
$2.5 \mathrm{nLS}$ & 525 & 225 & 262.5 & 18.8 & 3.1 \\
5nLS & 525 & 225 & 262.5 & 37.5 & 3.1 \\
\hline
\end{tabular}

Water, nanoCaCO${ }_{3}$, and $\mathrm{PC}$ added as suspension prepared by bath sonication 
7 days the sonicated mix exhibits higher strength than the plain 30FA sample by approximately $15 \%$. The stirred mix, on the other hand, exhibits a slightly lower strength than 30FA. This may be attributed to aggregation. Due to the high surface energy of the nanoparticles, the aggregates have the capacity to entrain air. As a result, the aggregates will act as weak points in the material and result in stress concentration. Similar to what is seen in the setting time results, sonication and PC treatment enhances the effect of the nanoparticles by improving dispersion. The exact mechanisms for the increase in strength at 7 days by the nanoCaCO${ }_{3}$ is unknown but can be the topic of future work.

The effect of higher additions of nanoCaCO 3 is determined and shown in Fig. 8. It is apparent that the most improvement is achieved with a $2.5 \%$ nanoC$\mathrm{aCO}_{3}$ addition, with a $20 \%$ increase in strength for $2.5 \mathrm{nLS}$ at 7 days compared to $30 \mathrm{FA}$. It is likely that the improvement in mechanical properties exhibited by mixes $1 \mathrm{nLS}$ and $2.5 \mathrm{nLS}$ are due to seeding and filler effects. It should be noted that the PC dosage is not constant throughout all mixes and this can affect strength results, with higher PC potentially leading to lower strength due to increased susceptibility to segregation and void formation. However, $2.5 \mathrm{nLS}$ exhibits the highest strength despite having a higher PC content. And 2.5nLS and 5nLS can be compared directly as they have the same PC content.

With the addition of $5 \%$ nanoCaCO 3 , the improvement in strength gain is less significant at 7 days and even detrimental at 1 day. Again, this can be tied to issues of dispersion. First, this is a relatively high

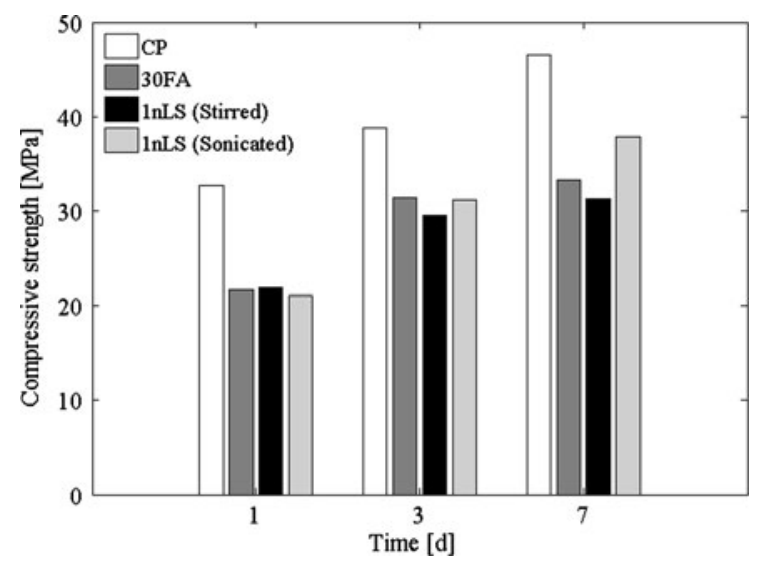

Fig. 7 Compressive strength gain: plain cement paste (CP) and $30 \%$ fly ash-cement pastes with $0 \%$ (30FA) and $1 \%$ nanoCaCO $3(1 \mathrm{nLS})$ addition [27] concentration of nanoparticles. Dispersion becomes increasingly more difficult as the concentration increases-due to the proximity of the particles they are more likely to reaggregate. Second, the PC concentration is limited to $8.3 \%$ by mass of nanoC$\mathrm{aCO}_{3}$ because a higher dosage would lead to segregation and bleeding of the CP. Dispersion results showed that the surface treatment is not sufficient at this concentration.

\subsubsection{SEM}

To better understand the compressive strength development of the various mixes and to complement the quantitative measure of dispersion obtained through spectroscopy, the morphology of 7 day pastes are examined through SEM. At least 15 images are captured at random locations on each sample. SEM images of plain CP and $30 \%$ fly ash-CPs with 2.5 and $5 \%$ nanoCaCO $\mathrm{Caddition}_{3}$ are presented in Fig. 9.

The plain $\mathrm{CP}$ has an apparently compact microstructure filled with hydration products, shown in Fig. 9a, where an abundance of fibrillar $\mathrm{C}-\mathrm{S}-\mathrm{H}$ and $\mathrm{CH}$ can be seen. Compared to the plain $\mathrm{CP}$, there are no marked differences in morphology in the $30 \%$ fly ash-CP with 2.5 and $5 \%$ nanoCaCO${ }_{3}$ addition (Fig. 9b, c, respectively), as can be resolved from SEM imaging. However, the compressive strength of the $2.5 \mathrm{nLS}$ samples at 7 days is notably greater than that of the 5nLS samples. Based on the SEM imaging technique utilized, no conclusions can be made on the effect of nanoCaCO $\mathrm{Ca}_{3}$ in altering the microstructure.

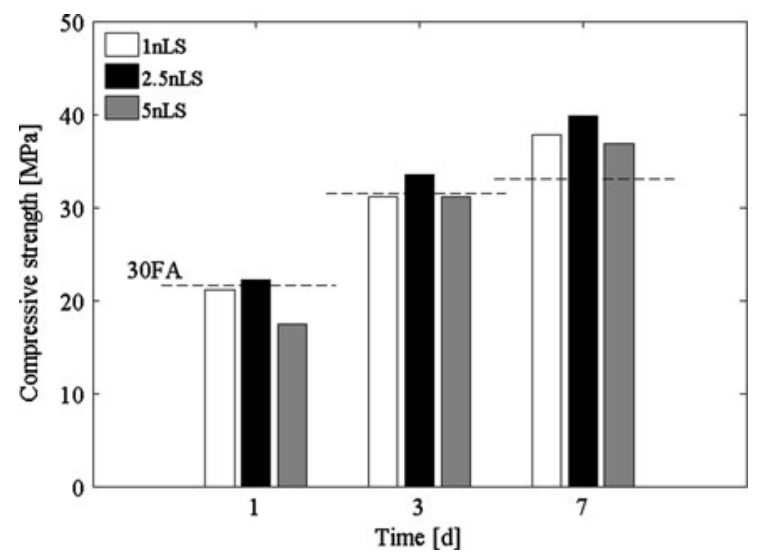

Fig. 8 Compressive strength gain of $30 \%$ fly ash-cement pastes with $1,2.5$, and $5 \%$ nanoCaCO 3 addition compared against $30 \%$ fly ash-cement paste (30FA) [27] 

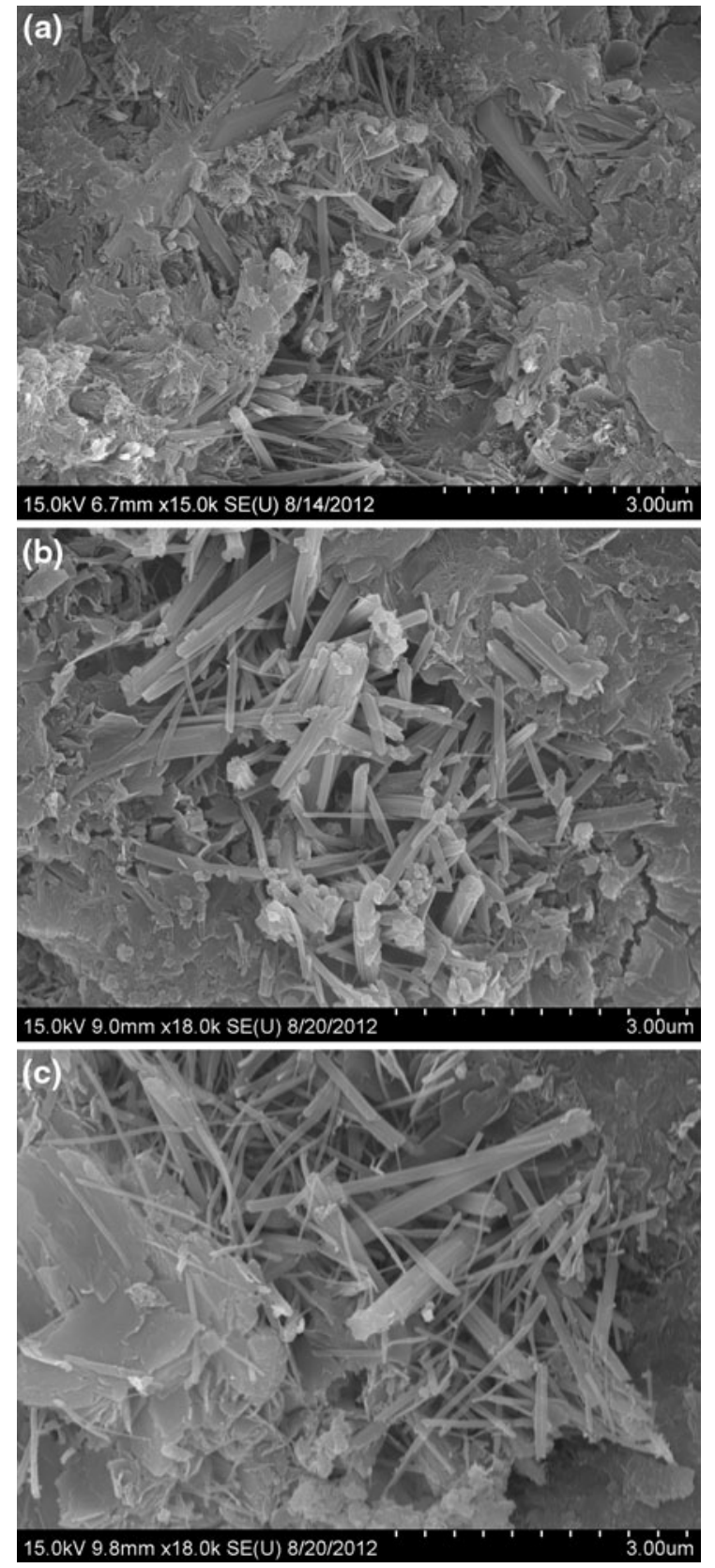

Fig. 9 SEM images of a plain cement paste and $30 \%$ fly ashcement pastes with b $2.5 \%$ and c $5 \%$ nanoCaCO 3 additions (Age $=7$ days) [27]

But the strength behavior can be at least partially explained by the difference in degree of aggregation that occurs in each of the two mixes.

A higher degree of aggregation occurs in the fly ash-CP with $5 \%$ nanoCaCO 3 , where a number of aggregates are spotted during SEM imaging, shown in
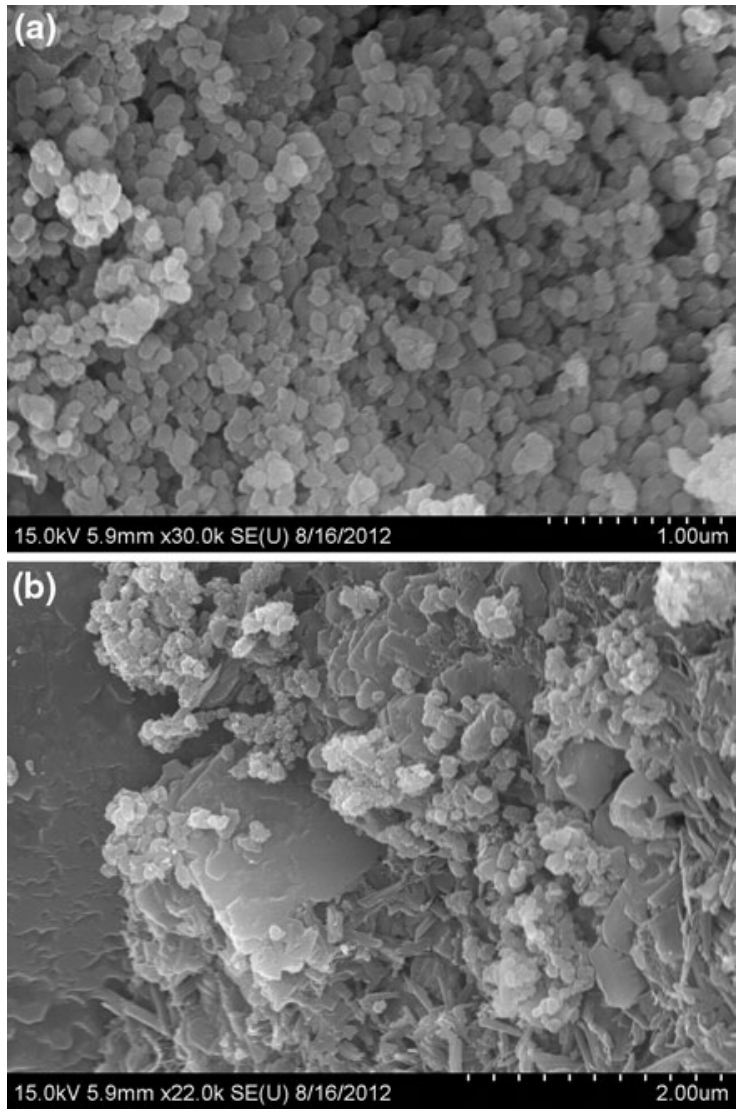

Fig. 10 SEM images of nanoCaCO 3 aggregates in $5 \%$ nanoCaCO $\mathrm{CO}_{3}$ fly ash-cement paste (Age $=7$ days) at a $30 \mathrm{k}$ and $\mathbf{b} 22 \mathrm{k}$ magnification [27]

Fig. 10. Visually, they are similar to the SEM images of the nanoCaCO 3 from an aqueous solution, Fig. 5 . EDS analysis also supports that they are $\mathrm{CaCO}_{3}$, as they exhibit $\mathrm{C} / \mathrm{Ca}$ ratios of about 0.7 . In comparison, hydrates exhibit either very low traces of carbon or none at all. Therefore, SEM images support that although the nanoCaCO${ }_{3}$ is aiding in densifying the microstructure in both systems, the lower compressive strength exhibited by the $5 \mathrm{nLS}$ samples may be attributed to the higher degree of aggregation. It also confirms that aggregated nanoparticles do not contribute to seeding; otherwise they would not be visible in the microstructure at 7 days.

\section{Conclusions}

In this study there was a focus on quantitatively measuring the dispersion of $\mathrm{CaCO}_{3}$ nanoparticles and tying it to their influence on the early-age properties of 
fly ash-CPs. From the surfactants studied, polycarboxylate superplasticizer is found to be the most effective at dispersing the nanoCaCO 3 in aqueous solution. It may be possible to further enhance its adsorption and dispersion capabilities by altering the polymer's architecture, which would improve the practical viability of the approach. Results indicate that sonication aids in the dispersion process by physically breaking up the nanoparticles. However, there appears to be a limit to the extent to which modifying the amplitude and duration can further improve dispersion and stability. With sonication and treatment with $\mathrm{PC}$, nanoCaCO 3 is found to accelerate setting and improve 7 days strength gain of fly ashCPs, as compared to stirred samples. At $2.5 \%$ addition, nanoCaCO $\mathrm{Ca}_{3}$ improves strength by $20 \%$ at 7 days. At $5 \%$ addition, there is a slightly negative effect due to aggregation, as indicated by the results of dispersion tests and SEM imaging.

Acknowledgments The authors would like to acknowledge the financial support from the Infrastructure Technology Institute at Northwestern University and Tennessee Valley Authority (TVA) and Oak Ridge Associated Universities (ORAU) (Award 105866). The second and fourth authors acknowledge funding from the National Science Foundation (DMR-1006391). SEM was performed in the NUANCE facility at Northwestern University, which is supported by the NSFMRSEC (DMR-1121262), Keck Foundation, and State of Illinois. The authors would also like to thank W.R. Grace, BASF, and Lafarge for providing materials.

\section{References}

1. Antaris AL, Seo J-WT, Green AA, Hersam MC (2010) Sorting single-walled carbon nanotubes by electronic type using nonionic, biocompatible block copolymers. ACS Nano 4(8):4725-4732

2. Arnold MS, Green AA, Hulvat JF, Stupp SI, Hersam MC (2006) Sorting carbon nanotubes by electronic structure using density differentiation. Nat Nano 1(1):60-65

3. Backfolk K, Lagerge S, Rosenholm JB (2002) The influence of stabilizing agents on the interaction between styrene/ butadiene latex and calcium carbonate: a calorimetric and a dynamic electrokinetic study. J Colloid Interface Sci 254(1):8-16

4. Bagheri A, Parhizkar T, Madani H, Raisghasemi AM (2012) The influence of different preparation methods on the aggregation status of pyrogenic nanosilicas used in concrete. Mater Struct 46(1-2):135-143

5. Bentz DP, Sato T, de la Varga I, Weiss WJ (2012) Fine limestone additions to regulate setting in high volume fly ash mixtures. Cem Concr Compos 34(1):11-17
6. Bjornstrom J, Chandra S (2003) Effect of superplasticizers on the rheological properties of cements. Mater Struct/ Materiaux et Constructions 36(264):685-692

7. Bonavetti VL, Rahhal VF, Irassar EF (2001) Studies on the carboaluminate formation in limestone filler-blended cements. Cem Concr Res 31(6):853-859

8. Camiletti J, Soliman AM, Nehdi ML (2012) Effects of nanoand micro-limestone addition on early-age properties of ultrahigh-performance concrete. Mater Struct 46(6):881-898

9. Chandra S, Bjornstrom J (2002) Influence of cement and superplasticizers type and dosage on the fluidity of cement mortars: Part I. Cem Concr Res 32(10):1605-1611

10. Chandra S, Bjornstrom J (2002) Influence of superplasticizer type and dosage on the slump loss of Portland cement mortars: Part II. Cem Concr Res 32(10):1613-1619

11. Chen J, He T, Wu W, Cao D, Yun J, Tan CK (2004) Adsorption of sodium salt of poly(acrylic) acid (PAANa) on nano-sized $\mathrm{CaCO}_{3}$ and dispersion of nano-sized $\mathrm{CaCO}_{3}$ in water. Colloids Surf A 232(2-3):163-168

12. Ferrari L, Kaufmann J, Winnefeld F, Plank J (2010) Interaction of cement model systems with superplasticizers investigated by atomic force microscopy, zeta potential, and adsorption measurements. J Colloid Interface Sci 347(1):15-24

13. Ferrari L, Kaufmann J, Winnefeld F, Plank J (2011) Multimethod approach to study influence of superplasticizers on cement suspensions. Cem Concr Res 41(10):1058-1066

14. Fu D, Wu S, He X, Ni J (2008) Preparation and property analysis of polyacrylate dispersant for calcium carbonate. Colloids Surf A 326(3):122-128

15. Ghrici M, Kenai S, Said-Mansour M (2007) Mechanical properties and durability of mortar and concrete containing natural pozzolana and limestone blended cements. Cem Concr Compos 29(7):542-549

16. Giraudeau C, D'Espinose De Lacaillerie J-B, Souguir Z, Nonat A, Flatt RJ (2009) Surface and intercalation chemistry of polycarboxylate copolymers in cementitious systems. J Am Ceram Soc 92(11):2471-2488

17. Green AA, Hersam MC (2011) Nearly single-chirality single-walled carbon nanotubes produced via orthogonal iterative density gradient ultracentrifugation. Adv Mater 23(19): 2185-2190

18. Green AA, Hersam MC (2009) Solution phase production of graphene with controlled thickness via density differentiation. Nano Lett 9(12):4031-4036

19. Greenwood R, Rowson N, Kingman S, Brown G (2002) A new method for determining the optimum dispersant concentration in aqueous grinding. Powder Technol 123(2-3): 199-207

20. Gurney L, Bentz DP, Sato T, Weiss WJ (2012) Using limestone to reduce set retardation in high volume fly ash mixtures: improving constructability for sustainability. Transp Res Rec 2290:139-146

21. Hanehara S, Yamada K (1999) Interaction between cement and chemical admixture from the point of cement hydration, absorption behaviour of admixture, and paste rheology. Cem Concr Res 29(8):1159-1165

22. Heikal M, El-Didamony H, Morsy MS (2000) Limestonefilled pozzolanic cement. Cem Concr Res 30(11):1827-1834

23. Hou P, Wang K, Qian J, Kawashima S, Kong D, Shah SP (2012) Effects of colloidal nanoSiO ${ }_{2}$ on fly ash hydration. Cem Concr Compos 34(10):1095-1103 
24. Irassar EF, González M, Rahhal V (2000) Sulphate resistance of type $\mathrm{V}$ cements with limestone filler and natural pozzolana. Cem Concr Compos 22(5):361-368

25. Ivanova NI, Shchukin ED (1993) Mixed adsorption of ionic and non-ionic surfactants on calcium carbonate. Colloids Surf A 76:109-113

26. Ji T (2005) Preliminary study on the water permeability and microstructure of concrete incorporating nano- $\mathrm{SiO}_{2}$. Cem Concr Res 35(10):1943-1947

27. Kawashima S (2012). Nanomodification of cementitious materials: Fresh state and early age. PhD Dissertation, Northwestern University, Evanston, IL: ProQuest/UMI, 2013, 196 pages (Publication No. AAT 3556618)

28. Kawashima S, Hou P, Corr DJ, Shah SP (2013) Modification of cement-based materials with nanoparticles. Cem Concr Compos 35:8-15

29. Kakali G, Tsivilis S, Aggeli E, Bati M (2000) Hydration products of $\mathrm{C}_{3} \mathrm{~A}, \mathrm{C}_{3} \mathrm{~S}$ and Portland cement in the presence of $\mathrm{CaCO}_{3}$. Cem Concr Res 30(7):1073-1077

30. Kim JH, Noemi N, Shah SP (2012) Effect of powder materials on the rheology and formwork pressure of selfconsolidating concrete. Cem Concr Compos 34(6):746-753

31. Kirby GH, Lewis JA (2004) Comb polymer architecture effects on the rheological property evolution of concentrated cement suspensions. J Am Ceram Soc 87(9):1643-1652

32. Kusters KA, Pratsinis SE, Thoma SG, Smith DM (1993) Ultrasonic fragmentation of agglomerate powders. Chem Eng Sci 48(24):4119-4127

33. Li C-Z, Feng N-Q, Li Y-D, Chen R-J (2005) Effects of polyethlene oxide chains on the performance of polycarboxylate-type water-reducers. Cem Concr Res 35(5):867-873

34. Lothenbach B, Le Saout G, Gallucci E, Scrivener K (2008) Influence of limestone on the hydration of Portland cements. Cem Concr Res 38(6):848-860

35. Mailvaganam NP, Rixom MR (1999) Chemical admixtures for concrete, 3rd edn. CRC Press, Boca Raton

36. Makar J (2011) The effect of SWCNT and other nanomaterials on cement hydration and reinforcement. In: Gopalakrishnan K, Birgisson B, Taylor P, Attoh-Okine N (eds) Nanotechnology in civil infrastructure. Springer, Berlin, pp 103-130

37. Mandzy N, Grulke E, Druffel T (2005) Breakage of $\mathrm{TiO}_{2}$ agglomerates in electrostatically stabilized aqueous dispersions. Powder Technol 160(2):121-126

38. Nyström R, Backfolk K, Rosenholm JB, Nurmi K (2003) Flocculation of calcite dispersions induced by the adsorption of highly cationic starch. Colloids Surf A 219(1-3):55-66

39. Ozyildirim C, Zegetosky C (2010) Exploratory investigation of nanomaterials to improve strength and permeability of concrete. Transp Res Rec J Transp Res Board 2142:1-8

40. Panya P, Arquero O-A, Franks GV, Wanless EJ (2004) Dispersion stability of a ceramic glaze achieved through ionic surfactant adsorption. J Colloid Interface Sci 279(1):23-35

41. Peng P, Garnier G (2012) Effect of cationic polyacrylamide on precipitated calcium carbonate flocculation: kinetics, charge density and ionic strength. Colloids Surf A 408:32-39

42. Péra J, Husson S, Guilhot B (1999) Influence of finely ground limestone on cement hydration. Cem Concr Compos 21(2):99-105
43. Plank J, Hirsch C (2007) Impact of zeta potential of early cement hydration phases on superplasticizer adsorption. Cem Concr Res 37(4):537-542

44. Plank J, Sachsenhauser B (2009) Experimental determination of the effective anionic charge density of polycarboxylate superplasticizers in cement pore solution. Cem Concr Res 39(1):1-5

45. Qing Y, Zenan Z, Deyu K, Rongshen C (2007) Influence of nano- $\mathrm{SiO}_{2}$ addition on properties of hardened cement paste as compared with silica fume. Constr Build Mater 21(3): $539-545$

46. Rosen MJ, Li F (2001) The adsorption of gemini and conventional surfactants onto some soil solids and the removal of 2-naphthol by the soil surfaces. J Colloid Interface Sci 234(2):418-424

47. Sato T, Beaudoin J (2011) Effect of nano- $\mathrm{CaCO}_{3}$ on hydration of cement containing supplementary cementitious materials. Adv Cem Res 23(1):33-43

48. Sato T, Diallo F (2010) Seeding effect of nano- $\mathrm{CaCO}_{3}$ on the hydration of tricalcium silicate. J Transp Res Board 2141:61-67

49. Seo J-WT, Green AA, Antaris AL, Hersam MC (2011) High-concentration aqueous dispersions of graphene using nonionic, biocompatible block copolymers. J Phys Chem Lett 2(9):1004-1008

50. Tyler TP, Shastry TA, Leever BJ, Hersam MC (2012) Narrow diameter distributions of metallic arc discharge single-walled carbon nanotubes via dual-iteration density gradient ultracentrifugation. Adv Mater 24(35):4765-4768

51. Uchikawa H, Hanehara S, Shirasaka T, Sawaki D (1992) Effect of admixture on hydration of cement, adsorptive behavior of admixture and fluidity and setting of fresh cement paste. Cem Concr Res 22(6):1115-1129

52. Uchikawa H, Sawaki D, Hanehara S (1995) Influence of kind and added timing of organic admixture on the composition, structure and property of fresh cement paste. Cem Concr Res 25(2):353-364

53. Winnefeld F, Becker S, Pakusch J, Götz T (2007) Effects of the molecular architecture of comb-shaped superplasticizers on their performance in cementitious systems. Cem Concr Compos 29(4):251-262

54. Zelić J, Jozić D, Krpan-Lisica D (2009) Synergistic action of a ternary system of portland cement-limestone-silica fume in concrete. In: Bittnar Z, Bartos PM, Němeček J, Šmilauer V, Zeman J (eds) Nanotechnology in construction 3. Springer, Berlin

55. Zingg A, Winnefeld F, Holzer L, Pakusch J, Becker S, Figi R, Gauckler L (2009) Interaction of polycarboxylate-based superplasticizers with cements containing different C3A amounts. Cem Concr Compos 31(3):153-162

56. Zingg A, Winnefeld F, Holzer L, Pakusch J, Becker S, Gauckler L (2008) Adsorption of polyelectrolytes and its influence on the rheology, zeta potential, and microstructure of various cement and hydrate phases. J Colloid Interface Sci 323(2):301-312 